\title{
Optical, Dosimeter, and Scintillation Properties of $\mathrm{MgB}_{2}$
}

\author{
Takayuki Yanagida, ${ }^{*}$ Yoshisuke Futami, ${ }^{1}$ Yutaka Fujimoto, ${ }^{2}$ Masanori Koshimizu, ${ }^{2}$ \\ Hiraku Ogino, ${ }^{3}$ Takumi Kato, Daisuke Nakauchi, and Go Okada \\ Nara Institute of Science and Technology, 8916-5 Takayama, Ikoma, Nara 630-0192, Japan \\ ${ }^{1}$ National Institute of Technology, Kumamoto College, \\ 2627 Hirayamashinmachi, Yatsushiro, Kumamoto 866-8501, Japan \\ ${ }^{2}$ Department of Applied Chemistry, Graduate School of Engineering, Tohoku University, \\ 6-6-07 Aoba, Aramaki, Aoba-ku, Sendai, Miyagi 980-8579, Japan \\ ${ }^{3}$ Department of Applied Chemistry, School of Engineering, The University of Tokyo, \\ 7-3-1 Hongo, Bunkyo-ku, Tokyo 113-8656, Japan
}

(Received December 13, 2015; accepted April 13, 2016)

Keywords: scintillator, superconducting material, $\mathrm{MgB}_{2}$, dosimeter, $\mathrm{X}$-ray

The optical, dosimeter, and scintillation properties of $\mathrm{MgB}_{2}$ were investigated for the first time. $\mathrm{MgB}_{2}$ was visibly black and showed $0.1-0.2 \%$ optical transmittance of wavelengths longer than $200 \mathrm{~nm}$. Under $280 \mathrm{~nm}$ excitation, an intense luminescence peak appeared around $500 \mathrm{~nm}$ with a fast decay time of $8.8 \mathrm{~ns}$. The temperature dependence of photoluminescence was examined in the range from 5 to $290 \mathrm{~K}$, and the $500 \mathrm{~nm}$ band disappeared at low temperatures. To evaluate dosimeter properties, the thermally stimulated luminescence (TSL) glow curve and the linearity between the TSL intensity and the X-ray irradiation dose were examined. As a result, $\mathrm{MgB}_{2}$ was found to exhibit good dosimeter properties. Finally, as a scintillation response, the X-ray-induced scintillation spectrum was evaluated and the emission peak at $470 \mathrm{~nm}$ was observed with a fast decay time of $11 \mathrm{~ns}$.

\section{Introduction}

$\mathrm{MgB}_{2}$ has attracted much attention because of its high-temperature superconductivity ${ }^{(1,2)}$ so that various studies have been conducted on it as a superconducting material. Despite numerous works on superconducting applications, the basic optical properties of $\mathrm{MgB}_{2}$ have not been studied at all. The interesting feature of $\mathrm{MgB}_{2}$ is that it has a chemical composition consisting of light elements, making it suitable for dosimeter application, which requires a biological tissue equivalency against $\mathrm{X}$ - and $\gamma$-ray exposure. Furthermore, ${ }^{10} \mathrm{~B}$ has a high interaction cross section with thermal neutrons and is potentially applicable to scintillators if detectable scintillation photons can be emitted. Therefore, $\mathrm{MgB}_{2}$ is potentially applicable to both types of ionizing radiation detectors.

Most personal dosimeters are composed of solid-state materials, such as $\mathrm{Al}_{2} \mathrm{O}_{3}$ in optically stimulated luminescence (OSL), ${ }^{(3)} \mathrm{LiF}$ in thermally stimulated luminescence (TSL), ${ }^{(4)}$ and Ag-doped phosphate glass in radiophotoluminescence (RPL). ${ }^{(5)}$ Although the physical processes and signal read out techniques are different in these dosimeters, the basic concept, the absorption and storage of the energy of ionizing radiation, and the release of the energy by ultraviolet (UV)-visible photon emissions are the same. On the other hand, scintillators are phosphors that convert a single high-

*Corresponding author: e-mail: t-yanagida@ms.naist.jp 
energy ionizing radiation into hundreds of visible photons, ${ }^{(6)}$ and scintillation detectors have been widely used in practical applications in, for example, medical, ${ }^{(7)}$ security, ${ }^{(8)}$ well-logging, ${ }^{(9)}$ and highenergy physics ${ }^{(10,11)}$ fields. Recent trends in solid-state radiation detectors focus on the development of scintillators for neutron detectors because of the shortage of ${ }^{3} \mathrm{He}$ gas. ${ }^{(12)}$ For neutron detectors, ${ }^{6} \mathrm{Li}$ - or ${ }^{10} \mathrm{~B}$-containing materials consisting of light elements are desirable for detecting neutrons and suppressing background $\gamma$-rays efficiently. We have expended effort to develop ${ }^{6} \mathrm{Li}^{(13-16)}$ - and ${ }^{10} \mathrm{~B}^{(17,18)}$-containing scintillators so far. $\mathrm{MgB}_{2}$ satisfies basic demands because it contains $\mathrm{B}$ in the chemical formula and the effective atomic number is low. Although the sample was fully black and the effective thickness was quite small, as described later, we could observe luminescence from $\mathrm{MgB}_{2}$.

On the basis of the conservation of energy, dosimeter and scintillation properties are complementary because the immediate release of energy (scintillation) and energy storage (dosimeter) are balanced. ${ }^{(19,20)}$ In other words, a dark scintillation material is a bright dosimeter material, and vice versa. Therefore, if the aim is radiation detection, these properties should be evaluated for one material. In this study, we focused on the investigation of basic properties under the irradiation of ionizing radiation of $\mathrm{MgB}_{2}$.

\section{Experimental Procedure}

A bulk sample of $\mathrm{MgB}_{2}$ was fabricated by a solid-state reaction of $\mathrm{Mg}$ and $\mathrm{B}$. A $\mathrm{Nb}$ tube filled with a powder mixture of $\mathrm{Mg}$ and $\mathrm{B}$ at a molar ratio of 1:2 was pressed into a tape form and both ends were mechanically closed. The sample was sintered at $800^{\circ} \mathrm{C}$ for $6 \mathrm{~h}$ in an evacuated quartz ampoule.

Optical transmittance and photoluminescence (PL) spectra were evaluated using JASCO V670 and Hamamatsu Quantaurus- $\tau$, respectively. In PL spectra, the excitation wavelength was $280 \mathrm{~nm}$. In order to investigate whether or not the superconductive temperature affects the luminescence properties, laser-excited PL spectra were collected from 5 to $290 \mathrm{~K}$. Then, PL decay time was investigated with the excitation wavelength of $280 \mathrm{~nm}$, which was the strongest emission wavelength of this instrument, and the monitoring wavelength of $500 \mathrm{~nm}$.

As a dosimeter property, the TSL glow curve and the linearity between the irradiated dose and the TLS intensity were evaluated by using Nanogray TL2000.(21) Then, the radioluminescence (RL) spectrum was collected using the $\mathrm{X}$-ray generator equipped with a $\mathrm{Cu}$ target supplied with $80 \mathrm{kV}$ bias voltage and $1 \mathrm{~mA}$ tube current. The emission was measured using the Andor DU-420BU2 charge-coupled device (CCD) spectrometer and the detailed explanation for the setup was previously reported. ${ }^{(22)}$ The scintillation decay time profile was investigated with our original setup, the pulse-X-ray-equipped afterglow characterization system. ${ }^{(23)}$ Except for the PL temperature dependence and TSL, all experiments were carried out at room temperature.

\section{Results and Discussion}

A sample of $\mathrm{MgB}_{2}$ is shown in Fig. 1. It had an area of approximately $7-8 \mathrm{~mm}^{2}$ and a thickness of $1 \mathrm{~mm}$. Under room light, it appeared dark and no transparency was observed. When it was illuminated by a UV lamp ( $254 \mathrm{~nm})$, we could see a green emission from the sample.

The transmittance spectrum of $\mathrm{MgB}_{2}$ is shown in Fig. 2. The transmittance was approximately $1 \%$ for near-infrared wavelengths and $0.1-0.2 \%$ for UV and visible wavelengths. The dip at 


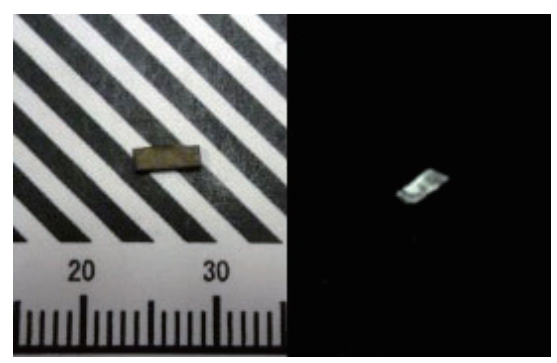

(a) (b)

Fig. 1. Sample of $\mathrm{MgB}_{2}$ under room (a) and UV (b) lights.

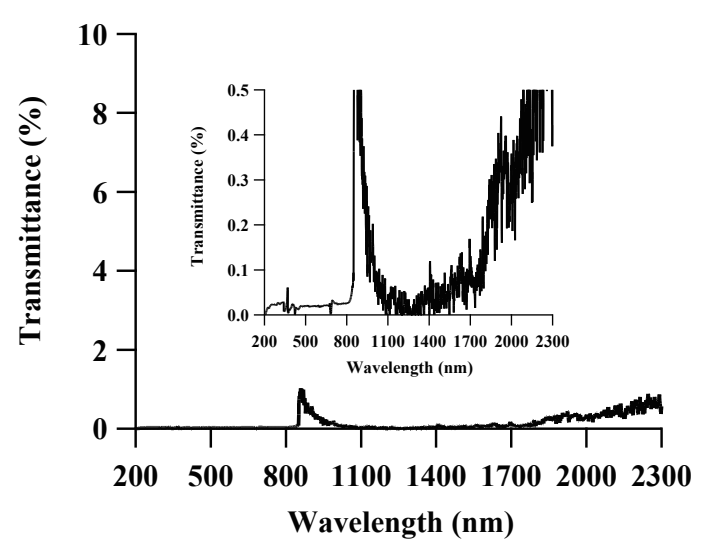

Fig. 2. Transmittance of $\mathrm{MgB}_{2}$.

around $800 \mathrm{~nm}$ was ascribed to an artifact of the instrument. By taking into account the $0.003 \%$ transparency at $500 \mathrm{~nm}$ and the sample thickness of $1 \mathrm{~mm}$, almost all the lights would be absorbed within a few hundred micrometers from the surface.

In Fig. 3, the PL spectrum of $\mathrm{MgB}_{2}$ under $280 \mathrm{~nm}$ excitation at room temperature is demonstrated. Strong emission was observed at around $500 \mathrm{~nm}$, which was consistent with the luminescence under UV excitation (Fig. 1) because the green wavelength is around $500 \mathrm{~nm}$. The temperature dependence of the PL is demonstrated in Fig. 4. A broad band peaking at $\sim 400 \mathrm{~nm}$ was observed. In addition, a sharp band was observed at $\sim 440 \mathrm{~nm}$ at low temperatures. These features are similar to the spectrum of grease, Apiezon-N, which was used to attach the sample to the copper sample holder. A notable feature in the temperature dependence is the appearance of the tail part at $450-600 \mathrm{~nm}$ at $290 \mathrm{~K}$. The wavelength range is consistent with that of the PL band in Fig. 3. This result strongly suggests that the emission band centered at $\sim 500 \mathrm{~nm}$ disappears at low temperatures, implying the involvement of some trap states.

The result of PL decay time profile monitoring at $500 \mathrm{~nm}$ under $280 \mathrm{~nm}$ excitation is shown in Fig. 5. Decay time was quite fast at $8.8 \mathrm{~ns}$. From the electronic structure of $\mathrm{MgB}_{2},{ }^{(24)}$ such luminescence was not expected. Although the origin of the emission was unclear, if the emission is caused by a contamination of transitional metals or rare-earth ions, some specific emission lines should be observed, and in most cases, the decay time should be slower except for the $\mathrm{Yb}^{3+}$ charge transfer emission. ${ }^{(25)}$ The other possibility is natural oxidation of the surface of the sample. However, $500 \mathrm{~nm}$ luminescence was not reported in $\mathrm{MgO}, \mathrm{B}_{2} \mathrm{O}_{3}$, or complex materials of $\mathrm{Mg}$ and $\mathrm{B}\left[\right.$ e.g., $\left.\mathrm{Mg}_{3}\left(\mathrm{BO}_{3}\right)_{2}\right]$. Taking into consideration these points, the origin of the luminescence could be ascribed to some types of defect or exciton, which sometimes show very fast luminescence (e.g., Ref. 26).

A typical TSL glow curve under 1 Gy exposure is shown in Fig. 6. The glow peak was successfully observed at around $370{ }^{\circ} \mathrm{C}$. By applying the initial rise method(27) for trap depth analysis, the trap depth was deduced to be $0.63 \mathrm{eV}$. This deep trap works well to avoid the fading in the TSL dosimeter. Then, the linearity between the irradiated dose and the TSL intensity is displayed in Fig. 7. From $20 \mathrm{mGy}$ to $20 \mathrm{~Gy}$, good linearity was observed. It was confirmed that $\mathrm{MgB}_{2}$ has potential for dosimeter application, although the origin of its emission should be clarified. 


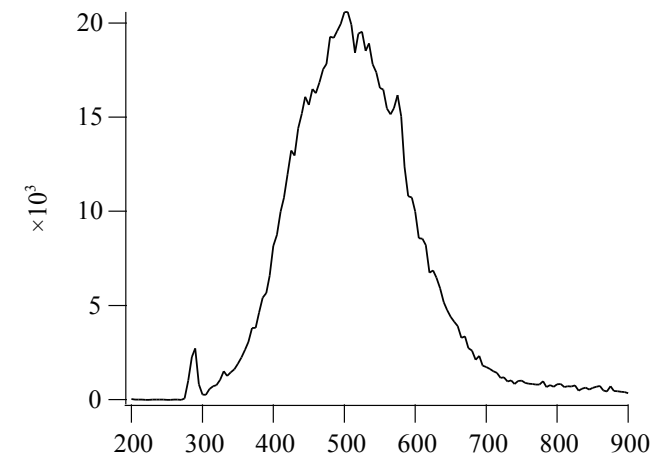

Fig. 3. PL spectrum of $\mathrm{MgB}_{2}$ under $280 \mathrm{~nm}$ excitation at room temperature.

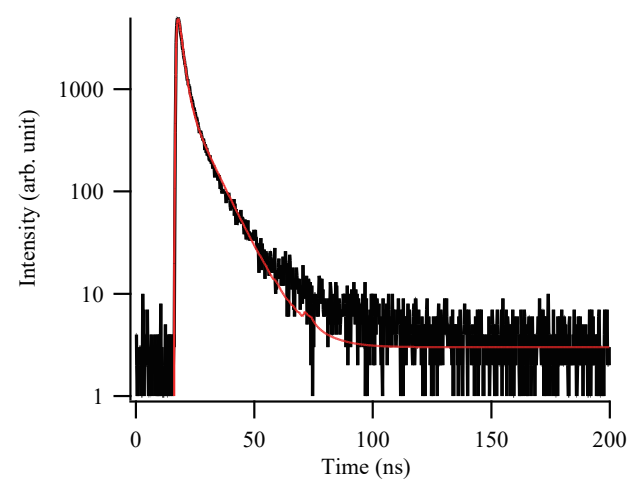

Fig. 5. (Color online) PL decay time profile of $\mathrm{MgB}_{2}$.

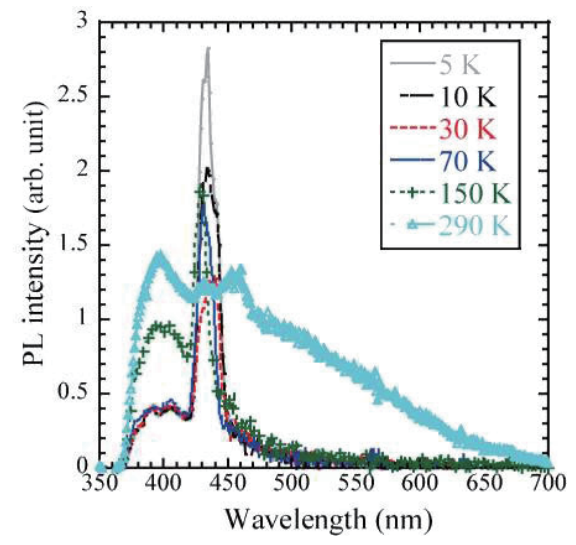

Fig. 4. (Color online) PL spectra of $\mathrm{MgB}_{2}$ under $325 \mathrm{~nm}$ laser excitation at various temperatures.

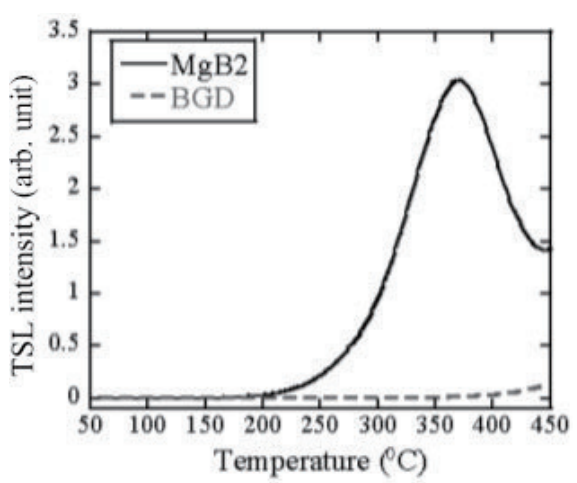

Fig. 6. TSL glow curve of $\mathrm{MgB}_{2}$ after $1 \mathrm{~Gy}$ exposure (solid line) and background (dotted line).

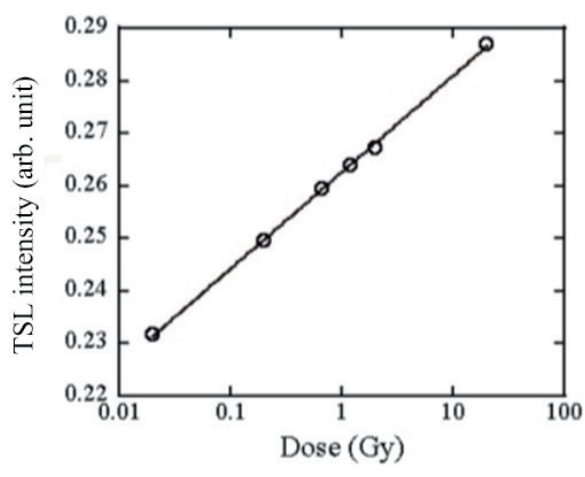

Fig. 7. Linearity between the irradiated dose (Gy) and TSL intensity (arbitrary unit).

The X-ray-induced RL spectrum at room temperature of $\mathrm{MgB}_{2}$ is exemplified in Fig. 8. The luminescence peak at $470 \mathrm{~nm}$ was detected while the intensity was not very high. The low emission intensity was explained by the stopping power of $\mathrm{MgB}_{2}$ against X-rays because the X-ray absorption probability was dominated by the density and the effective atomic number of 


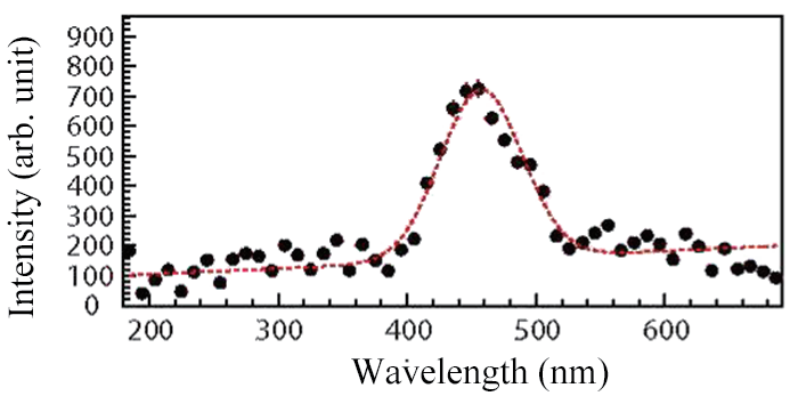

Fig. 8. (Color online) X-ray-induced $\mathrm{RL}$ of $\mathrm{MgB}_{2}$. The dotted line represents a fitting function.

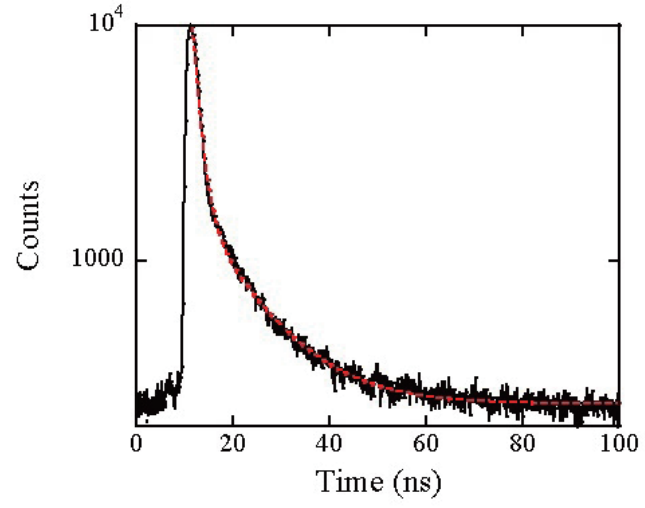

Fig. 9. (Color online) X-ray-induced scintillation decay time profile of $\mathrm{MgB}_{2}$ at room temperature.

the material. However, it was at least confirmed that $\mathrm{MgB}_{2}$ worked as a scintillator. In future prospects, a thermal-neutron-induced scintillation spectrum will be required for the evaluation of ${ }^{10} \mathrm{~B}$-enriched materials.

Figure 9 shows the X-ray-induced decay time profile at room temperature. Like the PL decay, very fast luminescence of $11 \mathrm{~ns}$ was observed, and no slow component was observed in this measurement time scale. If the scintillation light yield is improved, $\mathrm{MgB}_{2}$ will be applicable for high-counting-rate scintillation detectors.

\section{Conclusions}

The optical, TSL, and scintillation properties of $\mathrm{MgB}_{2}$ ceramic were investigated. $\mathrm{MgB}_{2}$ showed $0.1-0.2 \%$ transmittance for visible wavelengths and approximately $1 \%$ for near-infrared wavelengths. For PL, an intense emission peaking at $500 \mathrm{~nm}$ with a primary decay time of 8.8 ns was observed. The temperature dependence of PL was investigated and the band at $500 \mathrm{~nm}$ disappeared at low temperatures. As a dosimeter property, the TSL glow curve was studied. One glow peak appeared at $370{ }^{\circ} \mathrm{C}$ and this peak exhibited good linearity against X-ray exposure. Finally, the scintillation spectrum was evaluated and the luminescence peak was observed at 470 $\mathrm{nm}$. Although the sample was fully black and the effective thickness was quite small, throughout this work, $\mathrm{MgB}_{2}$ was confirmed to be applicable to radiation detectors as well as a superconducting material.

\section{Acknowledgements}

This research was supported by a Grant-in-Aid for Scientific Research (A) (26249147) and Grant-in-Aid for Research Activity Start-up (15H06409) from the Ministry of Education, Culture, Sports, Science and Technology (MEXT) of Japan. It was also partially supported by the Adaptable and Seamless Technology transfer Program (A-STEP) of the Japan Science and Technology (JST) Agency, KRF Foundation, Inamori Foundation, and the Cooperative Research Project of the Research Institute of Electronics, Shizuoka University. 


\section{References}

1 J. Nagamatsu, N. Nakagawa, T. Muranaka, Y. Zenitani, and J. Akimitsu: Nature 410 (2001) 63.

2 S. Souma Y. Machida, T. Sato, T. Takahashi, H. Matsui, S.-C. Wang, H. Ding, A. Kaminski, J. C. Campuzano, S. Sasaki, and K. Kadowaki: Nature 423 (2003) 65.

3 E. G. Yukihara and S. W. S. Mckeever: J. Appl. Phys. 100 (2006) 083512.

4 I. Tale, V. Tale, and P. Kulis: Radiat. Prot. Dosimetry 47 (1993) 107.

5 Y. Miyamoto, T. Ohno, Y. Takei, H. Nanto, T. Kurobori, T. Yanagida, A. Yoshikawa, Y. Nagashima, and T. Yamamoto: Radiat. Meas. 55 (2013) 72. doi:10.1016/j.radmeas.2012.10.018

6 T. Yanagida: Opt. Mater. 35 (2013) 1987.

7 T. Yanagida, A. Yoshikawa, Y. Yokota, K. Kamada, Y. Usuki, S. Yamamoto, M. Miyake, M. Baba, K. Sasaki, and M. Ito: IEEE Nucl. Trans. Sci. 57 (2010) 1492.

8 D. Totsuka, T. Yanagida, K. Fukuda, N. Kawaguchi, Y. Fujimoto, Y. Yokota, and A. Yoshikawa: Nucl. Instrum. Methods Phys. Res., Sect. A 659 (2011) 399.

9 T. Yanagida, Y. Fujimoto, S. Kurosawa, K. Kamada, H. Takahashi, Y. Fukazawa, M. Nikl, and V. Chani: Jpn. J. Appl. Phys. 52 (2013) 076401.

10 T. Ito, T. Yanagida, M. Sato, M. Kokubun, T. Takashima, S. Hirakuri, R. Miyawaki, H. Takahashi, K. Makishima, T. Tanaka, K. Nakazawa, T. Takahashi, and T. Honda: Nucl. Instrum. Methods Phys. Res., Sect. A 579 (2007) 239.

11 M. Kawaharada, S. Hong, M. M. Murashima, M. Kokubun, T. Itoh, K. Makishima, R. Miyawaki, H. Niko, T. Yanagida, T. Mitani, K. Nakazawa, K. Oonuki, T. Takahashi, K. Tamura, T. Tanaka, Y. Terada, Y. Fukazawa, N. Kawano, K. Kawashima, M. Ohno, K. Yamaoka, K. Abe, M. Suzuki, M. Tashiro, D. Yonetoku, and T. Murakami: Proc. SPIE 5501 (2004) 286.

12 R. T. Kouzes: The 3He Supply Problem, Technical Rpt. PNNL-18388, Pacific Northwest National Laboratory (2009).

13 T. Yanagida, Y. Fujimoto, K. Fukuda, N. Kawaguchi, K. Watanabe, A. Yamazaki, A. Uritani, and V. Chani: Opt. Mater. 35 (2013) 1449.

14 T. Yanagida, N. Kawaguchi, Y. Fujimoto, K. Fukuda, Y. Yokota, A. Yamazaki, K. Watanabe, J. Pejchal, A. Uritani, T. Iguchi, and A. Yoshikawa: Opt. Mater. 33 (2011) 1243.

15 K. Watanabe, T. Yanagida, K. Fukuda, A. Koike, T. Aoki, and A. Uritani: Sens. Mater. 27 (2015) 269.

16 K. Watanabe, Y. Kawabata, A. Yamazaki, A. Uritani, T. Iguchi, K. Fukuda, and T. Yanagida: Nucl. Instrum. Methods Phys. Res., Sect. A 802 (2015) 1.

17 Y. Fujimoto, T. Yanagida, Y. Yokota, N. Kawaguchi, K. Fukuda, D. Totsuka, K. Watanabe, A. Yamazaki, and A. Yoshikawa: Radiat. Meas. 46 (2011) 1506.

18 Y. Fujimoto, Y. Yokota, T. Yanagida, J. Pejchal, N. Kawaguchi, K. Fukuda, D. Totsuka, M. Nikl, and A. Yoshikawa: Nucl. Instrum. Methods Phys. Res., Sect. A 652 (2011) 238.

19 T. Yanagida: J. Lumin. 169 (2016) 544.

20 T. Yanagida, Y. Fujimoto, K. Watanabe, K. Fukuda, N. Kawaguchi, Y. Miyamoto, and H. Nanto: Radiat. Meas. 71 (2014) 162.

21 T. Yanagida, Y. Fujimoto, N. Kawaguchi, and S. Yanagida: J. Ceram. Soc. Jpn. 121 (2013) 988.

22 T. Yanagida, K. Kamada, Y. Fujimoto, H. Yagi, and T. Yanagitani: Opt. Mater. 35 (2013) 2480.

23 T. Yanagida, Y. Fujimoto, T. Ito, K. Uchiyama, and K. Mori: Appl. Phys. Express 7 (2014) 062401.

24 J. Kortus, I. I. Mazin, K. D. Belashchenko, V. P. Antropov, and L. L. Boyer: Phys. Rev. Lett. 86 (2011) 4656.

25 T. Yanagida, Y. Fujimoto, S. Kurosawa, K. Watanabe, H. Yagi, T. Yanagitani, V. Jary, Y. Futami, Y. Yokota, A. Yoshikawa, A. Uritani, T. Iguchi, and M. Nikl: Appl. Phys. Express 4 (2011) 1264.

26 M. Koshimizu, T. Yanagida, Y. Fujimoto, A. Yamazaki, K. Watanabe, A. Uritani, K. Fukuda, N. Kawaguchi, S. Kishimoto, and K. Asai: Appl. Phys. Express 6 (2013) 062601

27 S. W. S. Mckeever: Thermoluminescence of Solids (Cambridge University Press, Cambridge, 1985). 